\title{
BMJ Open Prevalence of uncontrolled hypertension in people with comorbidities in sub- Saharan Africa: a systematic review and meta-analysis
}

\author{
Shukri F Mohamed (D) , ${ }^{1,2,3}$ Olalekan A Uthman (D) , ${ }^{4}$ Martin K Mutua, ${ }^{5}$ G Asiki, ${ }^{2}$ \\ Mustapha S Abba, ${ }^{6}$ Paramjit Gill ${ }^{1}$
}

To cite: Mohamed SF, Uthman OA, Mutua MK, et al. Prevalence of uncontrolled hypertension in people with comorbidities in sub-Saharan Africa: a systematic review and meta-analysis. BMJ Open 2021;11:e045880. doi:10.1136/ bmjopen-2020-045880

- Prepublication history and additional supplemental material for this paper are available online. To view these files, please visit the journal online (http://dx.doi.org/10.1136/ bmjopen-2020-045880).

Received 18 October 2020 Accepted 11 November 2021

Check for updates

(c) Author(s) (or their employer(s)) 2021. Re-use permitted under CC BY-NC. No commercial re-use. See rights and permissions. Published by BMJ.

For numbered affiliations see end of article.

Correspondence to Ms Shukri F Mohamed; smohamed@aphrc.org

\section{ABSTRACT}

Background The burden of uncontrolled hypertension in sub-Saharan Africa (SSA) is high and hypertension is known to coexist with other chronic diseases such as kidney disease, diabetes among others. This is the first systematic review and meta-analysis to determine the burden of uncontrolled hypertension among patients with comorbidities in SSA.

Methods A comprehensive search was conducted on MEDLINE, Excerpta Medica Database (Embase) and Web of Science to identify all relevant articles published between 1 January 2000 and 17 June 2021. We included studies that reported on the prevalence of uncontrolled hypertension among people in SSA who report taking antihypertensive treatment and have another chronic condition. A random-effects meta-analysis was performed to obtain the pooled estimate of the prevalence of uncontrolled hypertension among patients with comorbid conditions while on treatment across studies in SSA. Results In all, 20 articles were included for metaanalyses. Eleven articles were among diabetic patients, five articles were among patients with HIV, two were among patients with stroke while chronic kidney disease and atrial fibrillation had one article each. The pooled prevalence of uncontrolled hypertension among patients with comorbidities was $78.6 \%$ (95\% Cl $71.1 \%$ to $85.3 \%)$; $1^{2} 95.9 \%$, varying from $73.1 \%$ in patients with stroke to $100.0 \%$ in patients with atrial fibrillation. Subgroup analysis showed differences in uncontrolled hypertension prevalence by various study-level characteristics Conclusion This study suggests a high burden of uncontrolled hypertension in people with comorbidities in SSA. Strategies to improve the control of hypertension among people with comorbidities are needed.

PROSPERO registration number CRD42019108218.

\section{INTRODUCTION}

Hypertension is an important risk factor for cardiovascular diseases and a leading contributor to death globally. ${ }^{1}$ An estimated 1.4 billion people have hypertension globally with three quarter $(75 \%)$ of this population living in low and middle-income countries. ${ }^{23}$ Worldwide trend analysis based on a large data
Strengths and limitations of this study

- A published comprehensive protocol was used to identify all available evidence without language restriction, reporting in accordance with Preferred Reporting Items for Systematic reviews and MetaAnalyses guidelines, search using multiple electronic databases, searching grey literature, contacting experts in the field for additional data sources to reduce study selection bias and heterogeneity test by subgroup analyses and sensitivity analyses.

- The prevalence of uncontrolled hypertension in some comorbidities such as atrial fibrillation and chronic kidney disease was reported in single studies.

- Most of the studies included in the meta-analysis were hospital-based studies that used non-random sampling procedures.

- There was substantial heterogeneity between the studies.

set from multiple studies conducted between 1975 and 2015 in 200 countries showed no change in global mean blood pressure, but a substantial downward trend in high-income countries and a rise in low and middle-income countries. ${ }^{4}$ Levels of hypertension awareness, treatment and control improved by $2.9 \%$ in high-income countries while in low-middleincome countries, negligible improvements in awareness, treatment and control were observed. ${ }^{34}$ Africa is one of the regions in the world with the highest rates of uncontrolled blood pressure..$^{5}$ In a systematic review and meta-analysis on hypertension in sub-Saharan Africa (SSA), Ataklte $e t a t^{6}$ reported a $(93 \%)$ high prevalence of uncontrolled hypertension (UHTN).

Hypertension often coexists with comorbidities such as chronic kidney disease, diabetes and hypercholesterolemia among others. ${ }^{7-10}$ These comorbidities could explain part of the inadequacy in blood pressure control. Some studies conducted in Europe and the 
USA found that patients with diabetes mellitus had a significantly increased risk of uncontrolled blood pressure. ${ }^{11} 12$ Another study conducted in the UK has shown that achieving optimal blood pressure control in patients with hypertension and type 2 diabetes produces an important decrease in the risks associated with diabetes. ${ }^{13}$

In recent years, public health efforts to promote prevention, awareness and treatment of hypertension in SSA have intensified, ${ }^{14-17}$ but hypertension control remains low. ${ }^{18-22}$ Despite several studies conducted on UHTN in people with comorbidities, pooled estimations of the burden are not available for comorbidities such as diabetes, dyslipidaemia, stroke, HIV, obesity atrial fibrillation. From a clinical perspective, it is important to understand why patients on treatment are not attaining optimal blood control and whether their pre-existing comorbidities contribute to the lack of control of blood pressure. Therefore, to inform policy, practice and the development of guidelines for hypertension for integrated care among patients with comorbid conditions, it is critical to understand the burden of UHTN in people with comorbidities. The purpose of this review is to summarise the evidence on and estimate the prevalence of UHTN in patients with comorbidities in SSA and to explore factors associated with UHTN in people with comorbidities.

\section{METHODS}

\section{Protocol and registration}

The protocol for this systemic review and meta-analysis was registered on the International Prospective Register of Systematic Reviews and published. ${ }^{23}$ The reporting was done according to the Preferred Reporting Items for Systematic reviews and Meta-Analyses (PRISMA) Protocols guidelines. ${ }^{24}$

\section{Search strategy}

We systematically searched MEDLINE via Ovid, Excerpta Medica Database (Embase) and Web of Science from 1 January 2000 to 17 June 2021. The search strategy included the following relevant terms: uncontrolled hypertension, hypertension, uncontrolled blood pressure, high blood pressure, a list of comorbidities and sub-Saharan Africa (detailed search strategy list is attached as supplement (online supplemental file S1). Additionally, the reference lists of the included studies were reviewed to identify other relevant studies.

\section{Eligibility criteria}

Studies were included if (1) they provided primary data on the prevalence of hypertension in accordance with the seventh report of the Joint National Committee among those who reported taking antihypertensive treatment and had a comorbid condition, (2) participants had been diagnosed with one of the comorbidities of interestdiabetes, dyslipidaemia, obesity, chronic kidney disease, stroke or transient ischaemic attack, coronary heart disease, heart failure, peripheral vascular disease, atrial fibrillation, depression and HIV (online supplemental table S1), (3) participants were 15 and above years, (4) the study was published in any language and (5) the study was conducted in an SSA. The following types of study designs were excluded: (1) case-control studies, commentaries, editorials, letters, qualitative studies and systematic reviews; (2) studies that included hypertension prevalence but did not report on the prevalence of hypertension among those on antihypertensive medication and (3) studies of pregnancy-related hypertension.

\section{Study selection}

Two researchers independently screened the titles and abstracts (SFM and MSA). Two researchers (SFM and MSA) also assessed full-text reviews of the articles independently for final inclusion. The reference lists of potentially relevant publications were manually searched for additional publications. Disagreements were resolved by consensus. For multinational studies, data were separated to show the estimate at the country level.

\section{Data items and collection process}

SFM and MSA independently screened the full texts of included studies. SFM extracted data from the selected studies and MSA checked the data for accuracy. A standardised data extraction table was created (table 1) and included the following data from all eligible articles: first author name, year of publication, language, country of the study, study design, sample size, study period, study setting, sampling method, the timing of data collection, data source, use of comorbidity-specific hypertension control cut-off, male proportion, age of participants (mean or median), type of comorbidity (diabetes, stroke, HIV, chronic kidney disease, atrial fibrillation) and main outcome of interest UHTN proportion or the data to cumpute it.

\section{Risk of bias in individual studies}

A tool developed by Hoy $e t a l^{25}$ for prevalence studies was adapted and used to assess the methodological quality of included studies by evaluating the extent to which they addressed bias in nine areas of internal and external validity (online supplemental table S2). Each of the nine areas was scored 1 if yes (high quality) and 0 if no (poor quality), and a total quality score was calculated by summing the individual scores. Total scores ranged from 0 to 9 , with higher scores indicating higher quality. Studies were then classified as having a low $(>8)$, moderate (6-8) or high ( $\leq 5)$ risk of bias. Two researchers (SFM and MKM) independently assessed each of the included publications and disagreements were resolved through discussion.

\section{Patient and public involvement}

This research was done without patient involvement. There was no involvement of patients or members of the public in the design, or conduct, or reporting or dissemination plans of this research. 


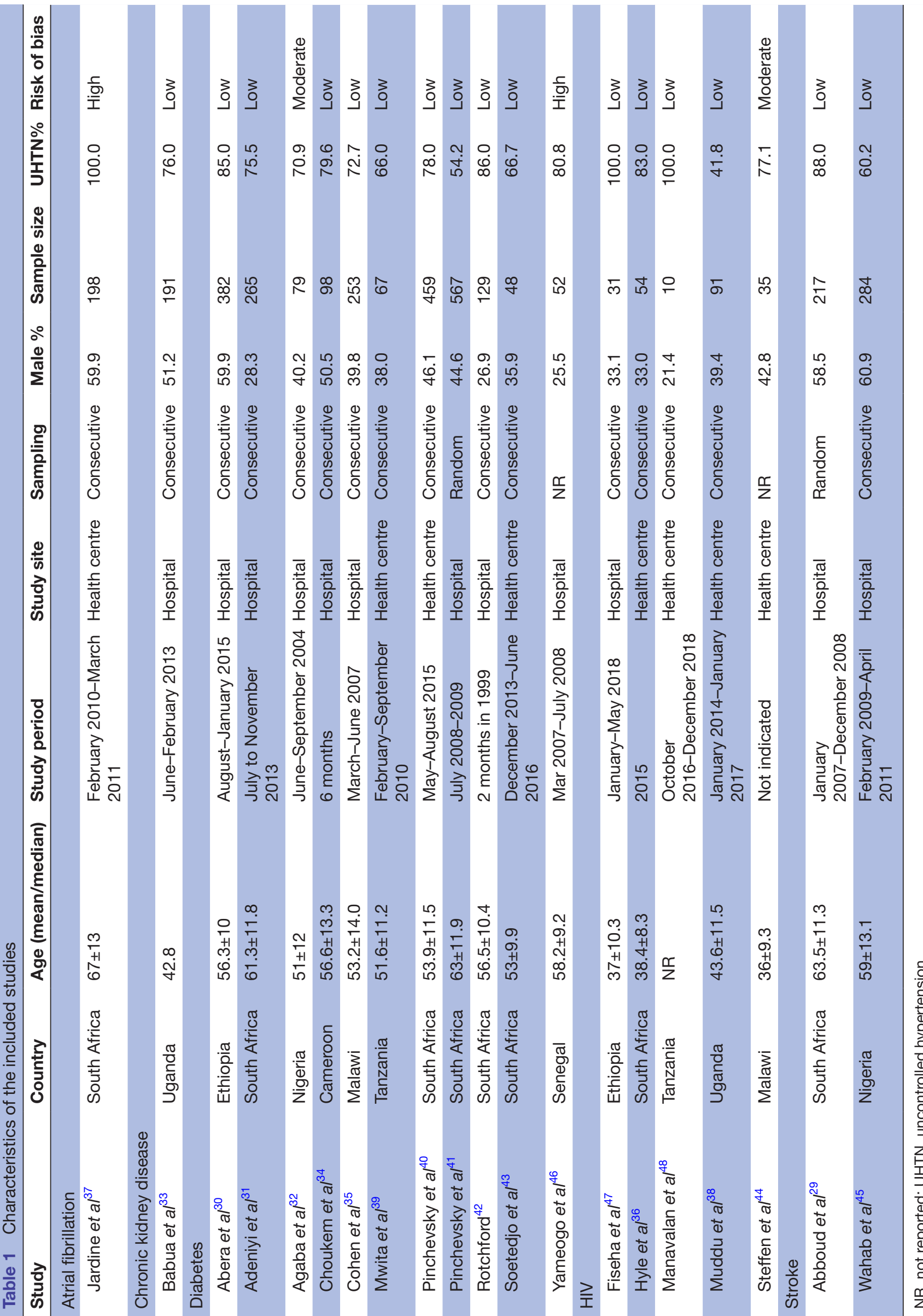




\section{Synthesis of results}

The statistical approach used in this meta-analysis followed the study protocol. ${ }^{23}$ Crude numerators and denominators from the individual studies were used to recalculate the study-specific unadjusted prevalence estimates. Variances of the study-specific estimates were stabilised using the double arcsine transformation to minimise the effect of studies with very small or very large prevalence estimates on the overall estimate (16), and then a random-effects meta-analysis was performed (17) to determine the pooled estimate of the prevalence of UHTN among patients with comorbidities overall and also among people with diabetes, HIV and stroke separately while on antihypertensive treatment across the included studies in SSA. Prevalence estimates were also summarised by comorbidities, publication year, sample size, study setting, sampling, risk of bias, gender proportionmean age and geographic regions.

Heterogeneity was explored using Cochrane's $Q$ and quantified by $\mathrm{I}^{2}$ statistics. ${ }^{26}{ }^{27}$ Subgroup analyses were performed based on the following: gender proportion of participants, patient comorbidities, study design, study setting, sample size, use of recommended comorbidityspecific blood pressure control cut-offs, countries, regions (Eastern, Western, Central, and Southern Africa) and by gross national income (GNI) were performed to identify the possible sources of heterogeneity. Sensitivity analyses were performed to assess the robustness of the findings by excluding studies with a high risk of bias.

Funnel plots and Egger asymmetry test were used to assess publication bias, with $\mathrm{p}<0.10$ considered to be statistically significant for publication bias. ${ }^{28}$ Inter-rater agreements between the researchers involved in study inclusion and those involved in the identification of risk of bias were assessed using $\kappa$ Cohen's coefficient (20).

All analyses were performed using 'metaprop' routine using StataSE V.16 (StataCorp LLC).

\section{RESULTS}

\section{Study selection}

From the electronic database search, 8492 records were identified. An additional 35 articles were identified through reference tracing and from other sources. After duplicate removal, 5610 remained for the title and abstract screening. After screening, we found 5085 records to be irrelevant and excluded them. The full texts of 525 articles and reports were retrieved and assessed for eligibility, resulting in the inclusion of 20 studies for the meta-analysis (figure 1). The inter-rater agreement for study selection was 0.77 .

\section{Study characteristics}

Table 1 and online supplemental table S3 provide detailed information on the included studies. In total, 3510 participants were included across 20 studies. Most of the studies were cross-sectional $(19,95 \%)$, in English (19, 95\%), hospital-based $(12,60 \%)$, used consecutive sampling

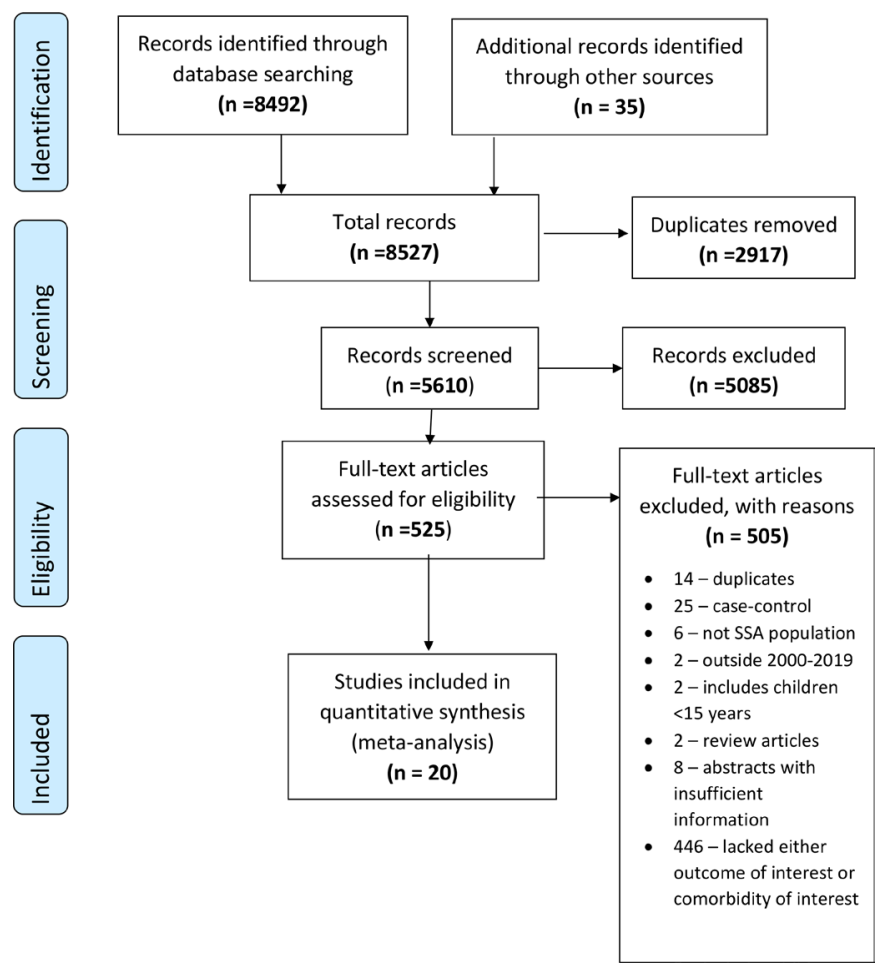

Figure 1 Study selection flow diagram.

$(16,80 \%)$ and prospectively collected data $(14,70 \%)$. The mean (SD) participant age from the 20 studies $^{29-48}$ providing this information was $56.8(0.12)$ years. Study sample sizes ranged from 10 to 567 participants. The proportion of male participants in the included studies was reported in all studies and it ranged from $21.4 \%$ to $60.9 \% .^{29-48}$ Of the included studies, $11^{30-32} 343539-4346$ reported on diabetes, five 36384448 reported on HIV, two ${ }^{29} 45$ reported on stroke and one each reported on chronic kidney disease ${ }^{33}$ and atrial fibrillation. ${ }^{37}$ None of the included studies reported on obesity, dyslipidaemia, coronary heart disease, heart failure, peripheral heart disease and depression.

\section{Risk of bias assessment}

The risk of bias was assessed in all included studies of the 20 included studies. Most studies were categorised as having some concern for bias with two $(10 \%)^{3746}$ studies being deemed to have high risk of bias. Two studies ${ }^{3244}(11.1 \%)$ had a moderate risk of bias, while 16 studies $^{29-31} 33-3638-43454748$ (80\%) had a low risk of bias. The inter-rater agreement for the risk of bias assessment was 0.65. Additional details on the domains assessed are included in the risk of bias summary table in the online supplemental table S2.

\section{Prevalence of UHTN among patients with comorbidities}

Twenty publications reported on UHTN among patients with comorbidities (table 1). The majority of the studies were from South Africa $(8,40 \%){ }^{29} 313637$ 40-43 Uganda, ${ }^{33} 38$ Nigeria, ${ }^{32}{ }^{45}$ Malawi $^{354}{ }^{44}$ Ethiopia $^{30} 47$ and Tanzania, ${ }^{39} 48$ had two $(10 \%)$ studies each while Senegal ${ }^{46}$ and Cameroon ${ }^{34}$ had one study $(5 \%)$ each. The reported 
prevalence of UHTN among people with comorbidities ranged from $41.8 \%$ (95\% CI $32.2 \%$ to $52.0 \%$ ) in Uganda to $100.0 \%$ (95\% CI $98.1 \%$ to $100.0 \%$ ) in South Africa. The pooled UHTN prevalence estimate in patients with comorbidities from the random-effects meta-analysis was $78.6 \%$ (95\% CI $71.1 \%$ to $85.3 \%$ ). Substantial heterogeneity $\left(\mathrm{I}^{2}=95.9 \% ; \mathrm{p}<0.0001\right)$ existed in the included studies (table 2). Absence of publication bias is suggested

Table 2 Meta-analysis results for the prevalence of uncontrolled hypertension in people with comorbidities in sub-Saharan Africa

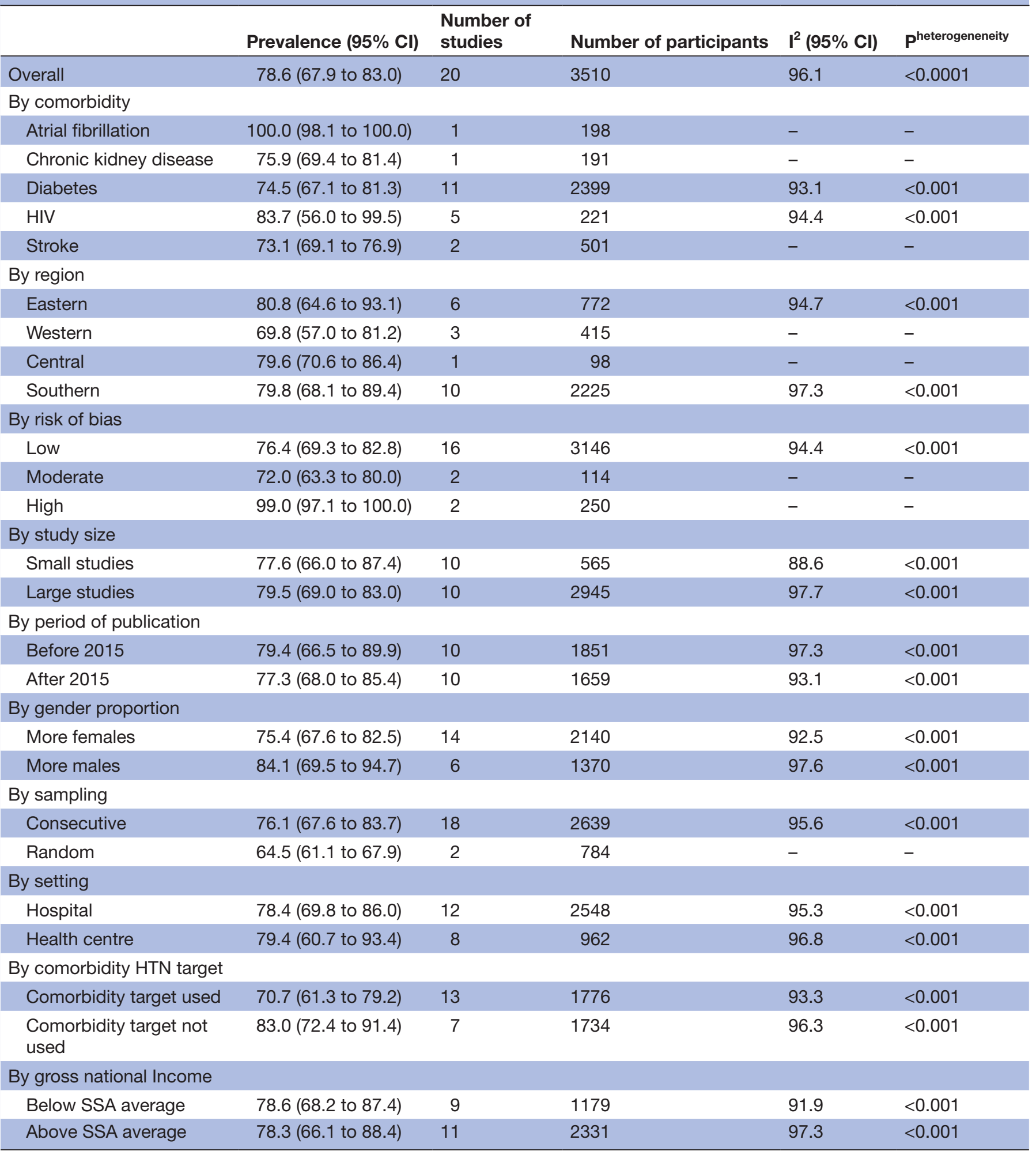

$\mathbf{P}^{\text {egger }}=0.381$

SSA, sub-Saharan Africa. 


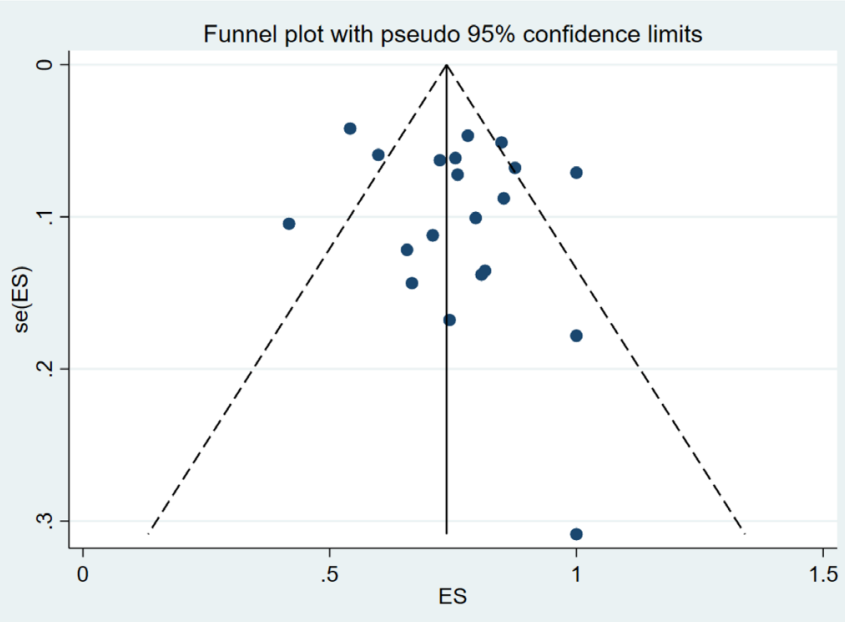

Figure 2 Funnel plot of the prevalence of uncontrolled hypertension in people with comorbidities in sub-Saharan Africa.

by the symmetrical visual inspection of the funnel plot, confirmed by the Egger's test $(\mathrm{p}<0.001)$ (figure 2$)$.

Subgroup analysis revealed differences in UHTN prevalence by comorbidity (figure 3). Adults with atrial fibrillation reported the highest UHTN estimate ( $100.0 \%$ (95\% CI $98.1 \%$ to $100.0 \%)$ ), followed by adults with HIV (83.7\% (95\% CI 56.0\% to 99.5\%)). The lowest pooled UHTN prevalence estimate was found in adults with stroke $(73.1 \%$ (95\% CI $69.1 \%$ to $76.9 \%))$. Pooled UHTN prevalence differed by geographic regions; studies conducted in the Eastern, Southern and Central region reported higher prevalence's $(80.8 \%$ (95\% CI $64.6 \%$ to $93.1 \%))$, $(79.8 \%$ (95\% CI $68.2 \%$ to $89.3 \%)$ ) and $(79.6 \%$ (95\% CI $70.6 \%$ to $86.4 \%)$ ), respectively, than studies conducted in the Western region $(69.8 \%$ (95\% CI $57.0 \%$ to $81.2 \%)$ ). Prevalence varied by sample size; large studies reported a slightly higher prevalence $(79.5 \%$ (95\% CI $69.1 \%$ to $88.2 \%)$ ) compared with small studies $(77.6 \%$ (95\% CI $66.0 \%$ to $87.4 \%)$ ) (table 2). Studies that used the recomended hypertension control value for each comorbidity reported lower pooled prevalence of UHTN (75.8 (95\% CI 66.4 to 84.1)) compared with those who did not use the recomemded comorbidity-specific blood pressure control value (83.0 (95\% CI 72.4 to 91.4)).

In the univariable analysis, heterogeneity was explained by being woman $(11.3 \%)$, risk of bias $(18.4 \%)$, by regions $(15.8 \%)$, comorbidities $(3.5 \%)$ and using target blood pressure (21.3\%) (online supplemental table S4). However, only comorbidities and risk of bias were significant at $10 \%$, and these were added to the multivariable meta-regression analysis. The results from the multivariable meta-regression were not statistically significant. Sensitivity analysis conducted by excluding studies that had high risk of bias from the analysis did not show any

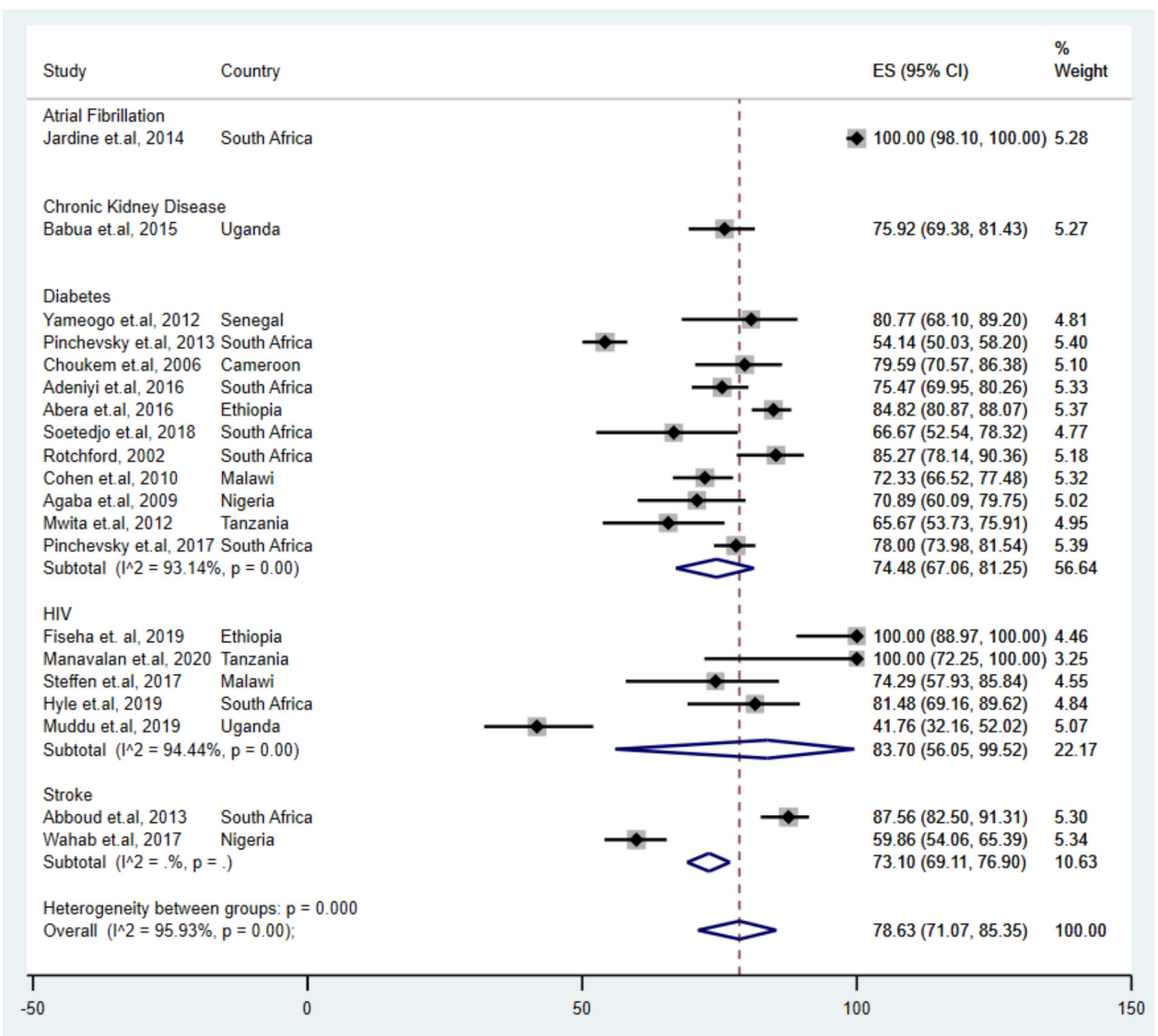

Figure 3 Pooled prevalence of uncontrolled hypertension in people with comorbidities in sub-Saharan Africa, by comorbidities. 


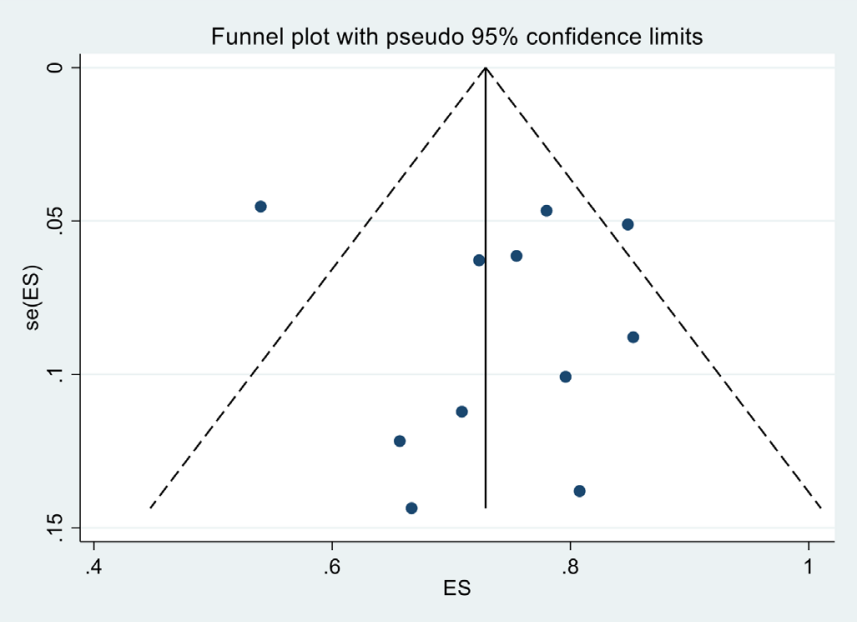

Figure 4 Funnel plot of the prevalence of uncontrolled hypertension in people with diabetes in sub-Saharan Africa.

influence on the robustness of the findings in the pooled analyses.

Sensitivity analysis done by excluding studies with high risk of bias from the analysis did not show any influence on the robustness of the findings in the pooled analysis.

\section{Prevalence of UHTN among patients with diabetes}

The prevalence of UHTN prevalence estimate among patients with diabetes was reported in 11 studies, ${ }^{30-32} 343539-4346$ with a total of 2399 participants. UHTN prevalence in this group ranged from $54 \%$ (95\% CI $50 \%$ to $58 \%$ ) to $85 \%$ (95\% CI $78 \%$ to $90 \%$ ), with a pooled estimate of $74.5 \%$ (95\% CI $67.1 \%$ to $81.3 \%$ ) (table 2). Substantial heterogeneity $\left(\mathrm{I}^{2}=93.1 \%\right.$; $\left.\mathrm{p}<0.001\right)$ was observed in the included studies (figure 2). Publication bias was not evident from the visual inspection of the funnel plot (figure 4).

Subgroup analysis revealed differences in UHTN prevalence among people with diabetes (table 3). There were differences noted by sample size; large studies reported a higher prevalence $(75.5 \%$ (95\% CI $67.1 \%$ to $81.3 \%)$ ) compared with small studies $(73.3 \%$ (95\% CI $68.2 \%$ to $79.3 \%$ )) (table 2 ). Pooled UHTN prevalence differed by geographic regions; studies conducted in the Eastern reported the highest pooled prevalence $(82.5 \%$ (95\% CI $80.4 \%$ to $87.1 \%)$ ) while studies conducted in the Southern region reported the lowest pooled prevalence $(72.5 \%$ (95\% CI $62.0 \%$ to $81.8 \%))$. Gender differences were also noted; studies with more male participants had higher pooled prevalence $(72.5 \%$ (95\% CI $64.4 \%$ to $79.9 \%)$ ) compared with studies with more female participants. Studies conducted after 2015 had higher pooled prevalence of UHTN among people with diabetes compared with studies conducted before 2015. Studies that used the recommended diabetes hypertension cut-off (BP $<130 / 85 \mathrm{~mm} \mathrm{Hg}$ ) to define blood pressure control reported lower UHTN prevalences compared with those who did not use the recommended hypertension control value. Studies that had below the average
SSA GNI reported a higher prevalence of UHTN (77.3 (95\% CI 69.7 to 84.2)) compared with studies with above the average SSA GNI (72.3 (95\% CI 61.0 to 82.3$)$ ).

In the univariable analysis, the use of the recomended hypertension control value for diabetes explained the most of the heterogeneity $(56.7 \%)$ observed while sampling explained $100 \%$ of the heterogeneity (online supplemental table S5). In the final multivariable model, the sampling method used was associated with UHTN and explained most of the heterogeneity.

\section{DISCUSSION}

To our knowledge, this is the first systematic review and meta-analysis on the pooled prevalence of UHTN among patients with comorbidities in SSA. Our findings indicate that more than three quarters of the hypertensive people with comorbidities have UHTN. These findings support the literature describing the challenges in controlling blood pressure among those on treatment and living with comorbidities while highlighting the fact that recognition of patient comorbidities should be a core aspect of the care and support offered to patients with hypertension.

The prevalence of UHTN varied with the type of comorbidity. The highest pooled UHTN prevalence estimate $(83.7 \%)$ was observed in people with HIV $(83.7 \%)$, chronic kidney disease $(75.9 \%)$ and diabetes $(74.5 \%)$. A systematic review and meta-analysis on the prevalence of hypertension among people with HIV showed that about $25 \%$ of people with HIV had hypertension. ${ }^{49}$ Also, important to note is that the majority of people living with HIV are in SSA. Similarly, a UK study found reduced risk associated with diabetes in people who achieved optimal blood pressure. ${ }^{13}$ Another study conducted in Kenya found that $80 \%$ of diabetic patients from rural and semiurban areas had hypertension. ${ }^{50}$ Since hypertension is common among people with comorbidities, there is need to focus on integrated care for comorbidities and hypertension. These findings support literature describing the challenge in blood pressure control among those on treatment and with comorbidities.

The high prevalence of UHTN in people with comorbidities is concerning and requires further understanding. There are several factors affecting UHTN among patients on treatment. Non-adherence to antihypertensive is an important cause of UHTN. A systematic review conducted by Abegaz et al found $45 \%$ of patients on antihypertensive were non-adherent to medications with a higher proportion $(84 \%)$ being among those with uncontrolled blood pressures. ${ }^{51}$ Barriers to adherence are mainly related to limited accessibility to medications, medication side effects, low perception of the risks involved with having uncontrolled blood pressure, out-of-pocket costs and pill burden due to comorbidities. Provider related factors also affect the UHTN rates. A study conducted by Rose et al concluded that inadequate treatment regimens are to blame for a majority of UHTN. ${ }^{8}$ Provider lack of adherence to hypertension guidelines in regards to dose 
Table 3 Meta-analysis results for the prevalence of uncontrolled hypertension in people with diabetes in sub-Saharan Africa

\begin{tabular}{|c|c|c|c|c|c|}
\hline & Prevalence $(95 \% \mathrm{Cl})$ & $\begin{array}{l}\text { Number of } \\
\text { studies }\end{array}$ & Number of participants & $\mathrm{I}^{2}(95 \% \mathrm{Cl})$ & $\mathbf{P}^{\text {heterogeneneity }}$ \\
\hline Overall & 74.5 (67.1 to 81.3$)$ & 11 & 2399 & 93.1 & $<0.001$ \\
\hline \multicolumn{6}{|l|}{ By region } \\
\hline Eastern & 82.5 (78.8 to 85.9$)$ & 2 & 449 & - & - \\
\hline Western & $75.0(67.1$ to 82.1$)$ & 2 & 131 & - & - \\
\hline Central & 79.6 (70.6 to 86.4$)$ & 1 & 98 & - & - \\
\hline Southern & 72.5 (62.0 to 81.8$)$ & 6 & 1721 & 94.9 & $<0.001$ \\
\hline \multicolumn{6}{|l|}{ By risk of bias } \\
\hline Low & 74.2 (65.8 to 81.9$)$ & 9 & 2268 & 94.4 & $<0.001$ \\
\hline Moderate & $70.9(60.1$ to 78.8$)$ & 1 & 79 & - & - \\
\hline High & 80.8 (68.1 to 89.2$)$ & 1 & 52 & - & - \\
\hline \multicolumn{6}{|l|}{ By study size } \\
\hline Small studies & 73.25 (66.8 to 79.3$)$ & 5 & 344 & 40.6 & 0.15 \\
\hline Large studies & 75.5 (64.82 to 84.8$)$ & 6 & 2055 & 96.4 & $<0.001$ \\
\hline \multicolumn{6}{|l|}{ By period of publication } \\
\hline Before 2015 & 72.9 (62.4 to 82.3$)$ & 4 & 1245 & 92.4 & $<0.001$ \\
\hline After 2015 & 78.0 (71.9 to 83.6$)$ & 7 & 1154 & 79.6 & $<0.001$ \\
\hline \multicolumn{6}{|l|}{ By gender proportion } \\
\hline More females & 72.5 (64.4 to 79.9$)$ & 9 & 1919 & 92.2 & $<0.001$ \\
\hline More males & 83.9 (80.4 to 87.1$)$ & 2 & 480 & - & - \\
\hline \multicolumn{6}{|l|}{ By sampling } \\
\hline Consecutive & 76.7 (72.3 to 80.9$)$ & 9 & 1780 & 75.2 & $<0.001$ \\
\hline Random & 54.1 (50.0 to 58.2) & 1 & 567 & - & - \\
\hline \multicolumn{6}{|l|}{ By setting } \\
\hline Hospital & 75.7 (66.0 to 84.3$)$ & 8 & 1825 & 94.9 & $<0.001$ \\
\hline Health centre & 71.6 (61.5 to 80.8$)$ & 3 & 574 & - & - \\
\hline \multicolumn{6}{|l|}{ By comorbidity HTN target } \\
\hline Comorbidity target used & 70.1 (57.4 to 81.5$)$ & 5 & 863 & 90.1 & $<0.001$ \\
\hline $\begin{array}{l}\text { Comorbidity target not } \\
\text { used }\end{array}$ & 78.2 (73.1 to 82.9$)$ & 6 & 1536 & 79.3 & $<0.001$ \\
\hline \multicolumn{6}{|l|}{ By gross national Income } \\
\hline Below SSA average & 77.3 (69.7 to 84.2$)$ & 5 & 852 & 81.1 & $<0.001$ \\
\hline Above SSA average & 72.3 (61.0 to 82.3$)$ & 6 & 1547 & 94.9 & $<0.001$ \\
\hline
\end{tabular}

$\mathrm{P}^{\text {egger }}<0.001$.

HTN, hypertension; SSA, sub-Saharan Africa.

escalation and use of multiple drug regimens are barrier to hypertension control. Chow et al revealed that the use of multiple drug regimens to treat hypertension was lower in low-income countries compared with the higher, upper middle or the lower middle-income countries. ${ }^{10}$

The prevalence of UHTN has declined significantly in studies published after 2015 compared with those published before 2015 probably because of adherence to the changing guidelines promoting tighter blood pressure control for people with comorbidities. However, despite the observed decline, the prevalence of UHTN among people with comorbidities is very high and needs further research to understand the interventions that can reduce the UHTN rate, so it can be adapted in other countries.

Our findings have the potential to inform public health strategies to reduce the burden of UHTN in SSA. Addressing the barriers identified is essential in achieving optimal blood pressure levels. The WHO's global target on hypertension control action plan recommends integrated care programmes for the management of hypertension and comorbidities, a recommendation supported by the results of the current study. ${ }^{1}$ 


\section{Strengths and limitations}

Strengths of our systematic review and meta-analysis include the use of a published comprehensive protocol ${ }^{23}$ to identify all available evidence without language restriction, reporting in accordance with PRISMA guidelines, search using multiple electronic databases, searching grey literature, contacting experts in the field for additional data sources to reduce study selection bias and heterogeneity test by subgroup analyses and sensitivity analyses.

This study should, however, be interpreted in the context of the following limitations. First, it is important to note that control of hypertension among those on treatment was not the main outcome of most of the included studies. Second, the prevalence of UHTN in some comorbidities such as atrial fibrillation and chronic kidney disease were reported in single studies probably because these conditions are understudied in SSA, thus limiting the generaliseability of such findings. Fourth, most of the studies included in the meta-analysis were hospitalbased studies $(60 \%)$ that used non-random sampling procedures $(80 \%)$. Therefore, population-based studies are warranted. Finally, we found substantial heterogeneity between the studies and conducted meta-regression analysis, which did not explain the heterogeneity. The lack of uniformity and variance in the blood pressure cut-off points for the different comorbidities may have resulted in this heterogeneity.

\section{CONCLUSION}

In conclusion, the prevalence of UHTN is high in people with comorbid conditions in SSA, particularly among people with diabetes. These findings strengthen the case for action to implement integrated care in the control of hypertension more effectively in African populations and other low-income and middle-income countries. Such efforts include improved access to blood pressure testing among people with comorbidities, strategies to improve adherence, reviewing treatment guidelines and training of healthcare workers in managing people with hypertension comorbidities and monitoring blood pressure control among all patients on treatment.

\section{Author affiliations}

${ }^{1}$ Academic Unit of Primary Care (AUPC) and the NIHR Global Health Research Unit on Improving Health in Slums, University of Warwick, Coventry, UK

${ }^{2}$ Health and Systems for Health, African Population and Health Research Center, Nairobi, Kenya

${ }^{3}$ Lown Scholars Program, Department of Global Health and Population, Harvard T.H. Chan School of Public Health, Boston, MA, USA

${ }^{4}$ Warwick-Centre for Applied Health Research and Delivery (WCAHRD), University of Warwick, Coventry, West Midlands, UK

${ }^{5}$ Data, Measurement and Evaluation unit, African Population and Health Rsearch Center, Nairobi, Kenya

${ }^{6}$ Division of Health Sciences, Warwick Medical School, University of Warwick, Coventry, West Midlands, UK

\section{Twitter Shukri F Mohamed @shukrifmohamed}

Acknowledgements The authors would like to thank Rishi Calleyachetty, (Assistant Professor, University of Warwick) and Ivy Chumo (Research Officer,
African Population and Health Research Center) for their initial support in the conceptualisation of this systematic review and meta-analysis. We also thank Samantha Johnson (Academic Support Librarian for Medicine, Life Sciences and Psychology, University of Warwick) for her guidance with the design of the initial literature search strategy.

Contributors SFM conceived the study. SFM, OAU, MKM, GA, MSA, and PG designed the search strategy. SFM and MSA conducted the searches, retrieved articles, screened abstract and title, and the full text of potentially relevant articles. SFM wrote the first draft of the manuscript. All authors critically revised the manuscript and contributed to subsequent iterations. SFM takes full responsibility for the work and /or the conduct of the study, had access to the data, and controlled the decision to publish.

Funding SFM, OAU and PG are supported by the National Institute for Health Research using Official Development Assistance (ODA) funding (NIHR Award ID: 16/136/87).

Disclaimer The views expressed in this publication are those of the author(s) and not necessarily those of the NHS, the National Institute for Health Research or the Department of Health and Social Care.

Competing interests None declared.

Patient consent for publication Not applicable.

Provenance and peer review Not commissioned; externally peer reviewed.

Data availability statement All data relevant to the study are included in the article or uploaded as supplementary information. No additional data available.

Supplemental material This content has been supplied by the author(s). It has not been vetted by BMJ Publishing Group Limited (BMJ) and may not have been peer-reviewed. Any opinions or recommendations discussed are solely those of the author(s) and are not endorsed by BMJ. BMJ disclaims all liability and responsibility arising from any reliance placed on the content. Where the content includes any translated material, BMJ does not warrant the accuracy and reliability of the translations (including but not limited to local regulations, clinical guidelines, terminology, drug names and drug dosages), and is not responsible for any error and/or omissions arising from translation and adaptation or otherwise.

Open access This is an open access article distributed in accordance with the Creative Commons Attribution Non Commercial (CC BY-NC 4.0) license, which permits others to distribute, remix, adapt, build upon this work non-commercially, and license their derivative works on different terms, provided the original work is properly cited, appropriate credit is given, any changes made indicated, and the use is non-commercial. See: http://creativecommons.org/licenses/by-nc/4.0/.

\section{ORCID iDs}

Shukri F Mohamed http://orcid.org/0000-0002-8693-1943

Olalekan A Uthman http://orcid.org/0000-0002-8567-3081

\section{REFERENCES}

1 World Health Organization,. Global Ncd target: reduce high blood pressure. World Health Organization, 2016.

2 Egan BM, Kjeldsen SE, Grassi G, et al. The global burden of hypertension exceeds 1.4 billion people: should a systolic blood pressure target below 130 become the universal standard? $J$ Hypertens 2019;37:1148-53.

3 Mills KT, Bundy JD, Kelly TN, et al. Global disparities of hypertension prevalence and control: a systematic analysis of population-based studies from 90 countries. Circulation 2016;134:441-50.

4 NCD Risk Factor Collaboration (NCD-RisC). Worldwide trends in blood pressure from 1975 to 2015: a pooled analysis of 1479 population-based measurement studies with $19 \cdot 1$ million participants. Lancet 2017;389:37-55.

5 NCD Risk Factor Collaboration (NCD-RisC). Worldwide trends in hypertension prevalence and progress in treatment and control from 1990 to 2019: a pooled analysis of 1201 population-representative studies with 104 million participants. Lancet 2021;398:957-80.

6 Ataklte F, Erqou S, Kaptoge S, et al. Burden of undiagnosed hypertension in sub-Saharan Africa: a systematic review and metaanalysis. Hypertension 2015;65:291-8.

7 World Health Organization. Counterfeit medicines: an update on estimates 15 November 2006, 2013.

8 Rose AJ, Berlowitz DR, Orner MB, et al. Understanding uncontrolled hypertension: is it the patient or the provider? J Clin Hypertens 2007;9): :937-43. 
9 Cushman WC, Ford CE, Cutler JA. Success and predictors of blood pressure control in diverse North American settings: the antihypertensive and lipid-lowering treatment to prevent heart attack trial (ALLHAT). J Clin Hypertens 2002;4:393-404.

10 Chow CK, Teo KK, Rangarajan S, et al. Prevalence, awareness, treatment, and control of hypertension in rural and urban communities in high-, middle-, and low-income countries. JAMA 2013;310:959-68.

11 Degli Esposti E, Di Martino M, Sturani A, et al. Risk factors for uncontrolled hypertension in Italy. J Hum Hypertens 2004;18:207-13.

12 Liu X, Song P. Is the association of diabetes with uncontrolled blood pressure stronger in Mexican Americans and blacks than in whites among diagnosed hypertensive patients? Am J Hypertens 2013;26:1328-34.

13 U. K. Prospective Diabetes Study Group. Tight blood pressure control and risk of macrovascular and microvascular complications in type 2 diabetes: UKPDS 38. UK prospective diabetes Study Group. BMJ 1998;317:703-13.

14 Cappuccio FP, Kerry SM, Micah FB, et al. A community programme to reduce salt intake and blood pressure in Ghana [ISRCTN88789643]. BMC Public Health 2006;6:13.

15 Oti SO, van de Vijver S, Gomez GB, et al. Outcomes and costs of implementing a community-based intervention for hypertension in an urban slum in Kenya. Bull World Health Organ 2016;94:501-9.

16 van de Vijver S, Oti SO, Gomez GB, et al. Impact evaluation of a community-based intervention for prevention of cardiovascular diseases in the slums of Nairobi: the scale-up study. Glob Health Action 2016;9:30922.

17 Ozoemena EL, Iweama CN, Agbaje OS, et al. Effects of a health education intervention on hypertension-related knowledge, prevention and self-care practices in Nigerian retirees: a quasiexperimental study. Arch Public Health 2019;77:23.

18 Mohamed SF, Mutua MK, Wamai R, et al. Prevalence, awareness, treatment and control of hypertension and their determinants: results from a national survey in Kenya. BMC Public Health 2018;18:1219.

19 Monakali S, Ter Goon D, Seekoe E, et al. Prevalence, awareness, control and determinants of hypertension among primary health care professional nurses in eastern Cape, South Africa. Afr J Prim Health Care Fam Med 2018;10:e1-e5.

20 Guwatudde D, Mutungi G, Wesonga R, et al. The epidemiology of hypertension in Uganda: findings from the National noncommunicable diseases risk factor survey. PLoS One 2015;10:e0138991

21 Mayige M, Kagaruki G. Tanzania steps survey report, 2013.

22 Msyamboza KP, Kathyola D, Dzowela T, et al. The burden of hypertension and its risk factors in Malawi: nationwide populationbased steps survey. Int Health 2012;4:246-52.

23 Mohamed SF, Uthman OA, Caleyachetty R, et al. Uncontrolled hypertension among patients with comorbidities in sub-Saharan Africa: protocol for a systematic review and meta-analysis. Syst Rev 2020;9:16.

24 Moher D, Liberati A, Tetzlaff J, et al. Preferred reporting items for systematic reviews and meta-analyses: the PRISMA statement. PLoS Med 2009;6:b2535.

25 Hoy D, Brooks P, Woolf A, et al. Assessing risk of bias in prevalence studies: modification of an existing tool and evidence of interrater agreement. J Clin Epidemiol 2012;65:934-9.

26 Higgins JPT, Thompson SG. Quantifying heterogeneity in a metaanalysis. Stat Med 2002;21:1539-58.

27 Higgins JPT, Thompson SG, Deeks JJ, et al. Measuring inconsistency in meta-analyses. BMJ 2003;327:557-60.

28 Egger M, Davey Smith G, Schneider M, et al. Bias in meta-analysis detected by a simple, graphical test. BMJ 1997;315:629-34.

29 Abboud H, Labreuche J, Arauz A, et al. Demographics, socioeconomic characteristics, and risk factor prevalence in patients with non-cardioembolic ischaemic stroke in low- and middle-income countries: the optic registry. Int J Stroke 2013;8 Suppl A100:4-13.

30 Abera $\mathrm{H}$, Woldemichael M. Pattern of antihypertensive therapy among diabetic HYPERTEN- SIVE patients in ZEWDITU Memorial Hospital, Addis ABABA. Ethiop Med J 2016;54:77-82.
31 Adeniyi OV, Yogeswaran P, Longo-Mbenza B, et al. Uncontrolled hypertension and its determinants in patients with concomitant type 2 diabetes mellitus (T2DM) in rural South Africa. PLoS One 2016;11:e0150033.

32 Agaba El, Puepet FH, Ugoya SO, et al. Chronic kidney disease screening and renoprotection in type 2 diabetes. Ann Afr Med 2009;8:52-4.

33 Babua C, Kalyesubula R, Okello E, et al. Cardiovascular risk factors among patients with chronic kidney disease attending a tertiary hospital in Uganda. Cardiovasc J Afr 2015;26:177-80.

34 Choukem SP, Kengne AP, Dehayem YM, et al. Hypertension in people with diabetes in sub-Saharan Africa: revealing the hidden face of the iceberg. Diabetes Res Clin Pract 2007;77:293-9.

35 Cohen DB, Allain TJ, Glover S, et al. A survey of the management, control, and complications of diabetes mellitus in patients attending a diabetes clinic in Blantyre, Malawi, an area of high HIV prevalence. Am J Trop Med Hyg 2010;83:575-81.

36 Hyle EP, Bekker L-G, Martey EB, et al. Cardiovascular risk factors among ART-experienced people with HIV in South Africa. J Int AIDS Soc 2019;22:e25274.

37 Jardine RM, Fine J, Obel IWP. A survey on the treatment of atrial fibrillation in South Africa. S Afr Med J 2014;104:623-7.

38 Muddu M, Tusubira AK, Sharma SK, et al. Integrated hypertension and HIV care cascades in an HIV treatment program in eastern Uganda: a retrospective cohort study. J Acquir Immune Defic Syndr 2019;81:552-61.

39 Mwita JC, Mugusi F, Lwakatare J, et al. Hypertension control and other cardiovascular risk factors among diabetic patients at Muhimbili national Hospital, Tanzania. East Afr J Public Health 2012;9:70-3.

40 Pinchevsky Y, Butkow N, Chirwa T, et al. Treatment gaps found in the management of type 2 diabetes at a community health centre in Johannesburg, South Africa. J Diabetes Res 2017;2017:1-6.

41 Pinchevsky Y, Butkow N, Raal FJ, et al. The implementation of guidelines in a South African population with type 2 diabetes. Journal of Endocrinology, Metabolism and Diabetes of South Africa 2013;18:154-8

42 Rotchford AP, Rotchford KM. Diabetes in rural South Africa--an assessment of care and complications. S Afr Med J 2002;92:536-41.

43 Soetedjo NNM, McAllister SM, Ugarte-Gil C, et al. Disease characteristics and treatment of patients with diabetes mellitus attending government health services in Indonesia, Peru, Romania and South Africa. Trop Med Int Health 2018;23:1118-28.

44 Steffen H, Phiri S, Heller T, et al. [PP.01.21] Hypertension prevalence, awareness, treatment and control in ambulatory treatment-naïve hiv infected patients in lilongwe, malawi. $J$ Hypertens 2017;35:e98.

45 Wahab KW, Kolo PM, Salawu FK, et al. Blood pressure control among hypertensive stroke survivors in Nigeria. J Stroke Cerebrovasc Dis 2017;26:1222-7.

46 Yaméogo NVet al. Control of cardiovascular risk in black Africans with type 2 diabetes in Senegal. Cardiovasc J Afr 2012;23:270-2.

47 Fiseha T, Belete AG, Dereje H, et al. Hypertension in HIV-infected patients receiving antiretroviral therapy in northeast Ethiopia. Int $J$ Hypertens 2019;2019:1-7.

48 Manavalan P, Madut DB, Hertz JT, et al. Hypertension burden and challenges across the hypertension treatment cascade among adults enrolled in HIV care in northern Tanzania. $J$ Clin Hypertens 2020;22:1518-22.

49 Xu Y, Chen X, Wang K. Global prevalence of hypertension among people living with HIV: a systematic review and meta-analysis. J Am Soc Hypertens 2017;11:530-40.

50 Githinji GG, Hussein AA, Kimani T, et al. Prevalence of diabetes and co-morbidities in five rural and semi-urban Kenyan counties, 2010-2015. Int J Diabetes Dev Ctries 2018;38:243-8.

51 Abegaz TM, Shehab A, Gebreyohannes EA, et al. Nonadherence to antihypertensive drugs: a systematic review and meta-analysis. Medicine 2017;96:e5641. 